\title{
Disección aórtica subaguda: correlación ecotomográfica
}

Mario Vargas Galgani

Servicio de Cardiología, Imagen cardíaca. Máster de ETE y Cardiorresonancia. Clínica San Felipe. Lima. Perú

\section{Correspondencia}

Mario Vargas Galgani

Correo: mariovar@hotmail.com

Recibido: 04/01/2020

Aceptado: 05/01/2020

En línea: 02/03/2020

Citar como: Vargas-Galgani M. Disección aórtica subaguda: correlación ecotomográfica. Rev Ecocar Pract (RETIC). 2020 (Mar); 3 (1): 27-30. doi: 10.37615/retic. v3n1a7.

Cite this as: Vargas-Galgani M. Subacute aortic dissection: echo and tomographic correlation. Rev Ecocar Pract (RETIC). 2020 (Mar); 3 (1): $27-30$. doi: 10.37615/ retic.v3n1a7.

\section{Palabras clave \\ $\triangleright$ Disección crónica \\ $\triangleright$ Aorta \\ $\triangleright$ Hematoma intramural}

Keywords

$\triangleright$ Chronic dissection

$\triangleright$ Aorta

$\triangleright$ Intramural hematoma

\section{Presentación del caso}

Se trata de un paciente adulto octogenario conocido por hipertensión crónica con cardiopatía hipertensiva, portador de marcapasos bicameral, insuficiencia aórtica y derrame pericárdico.

Cuatro semanas antes de ser visto, mientras se encontraba fuera de su país, presentó un dolor torácico agudo que fue catalogado como síndrome coronario y requirió un stent en arteria coronaria derecha aproximadamente 15 horas tras el inicio del dolor.

En una ecocardiografía transtorácica (ETT) de control, estando asintomático y ya en su país de procedencia (Lima), se detecta dilatación de la raíz aórtica con hematoma intramural en pared anterior que se extendía $3 \mathrm{~cm}$ distal con flujo de vaivén en su origen (Vídeo 1). En la Figura 1 se observa el hematoma intramural evaluado mediante modo M. También se detectaron los hallazgos habituales del paciente: hipertrofia leve del ventrículo izquierdo, derrame pericárdico, insuficiencia aórtica y no se encontraron trastornos segmentarios de motilidad (Figura 2, Vídeo 3). Haciendo un mapeo meticuloso desde paraesternal derecho alto, se encuentra otro flap en la aorta ascendente distal (Vídeo 4). No se tuvo buena ventana supraesternal para evaluar el arco aórtico, y la aorta abdominal en la vista subcostal no tenía flap de disección.
Se realizó de urgencia una tomografía computarizada (TC) con contraste que demostró ambas lesiones descritas por la ecocardiografía, refiriéndose el paciente para cirugía torácica y corrección quirúrgica (Figura 3, Vídeo 5 y Vídeo 6).

\section{Estudio por imagen}

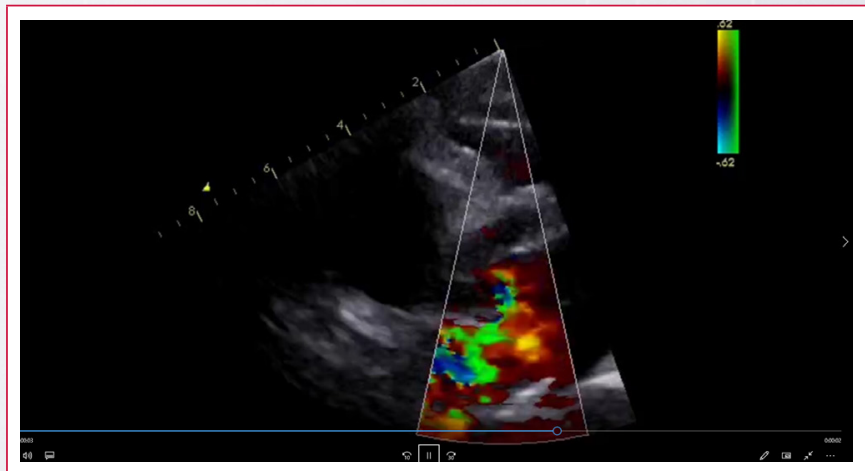

Vídeo 1. Vista paraesternal de eje largo que muestra hematoma intramural, flujo de vaivén en su origen e insuficiencia aórtica 


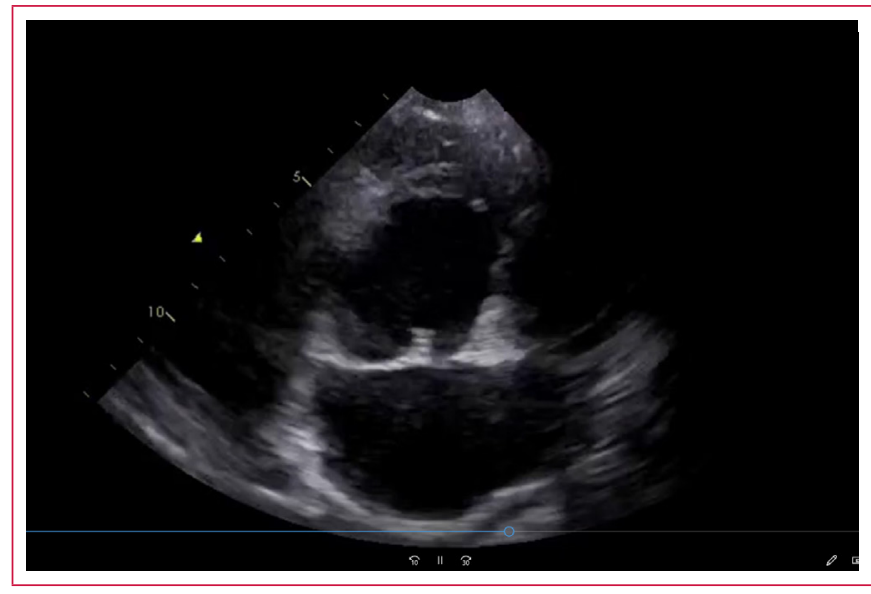

Vídeo 2. Hematoma intramural en eje corto

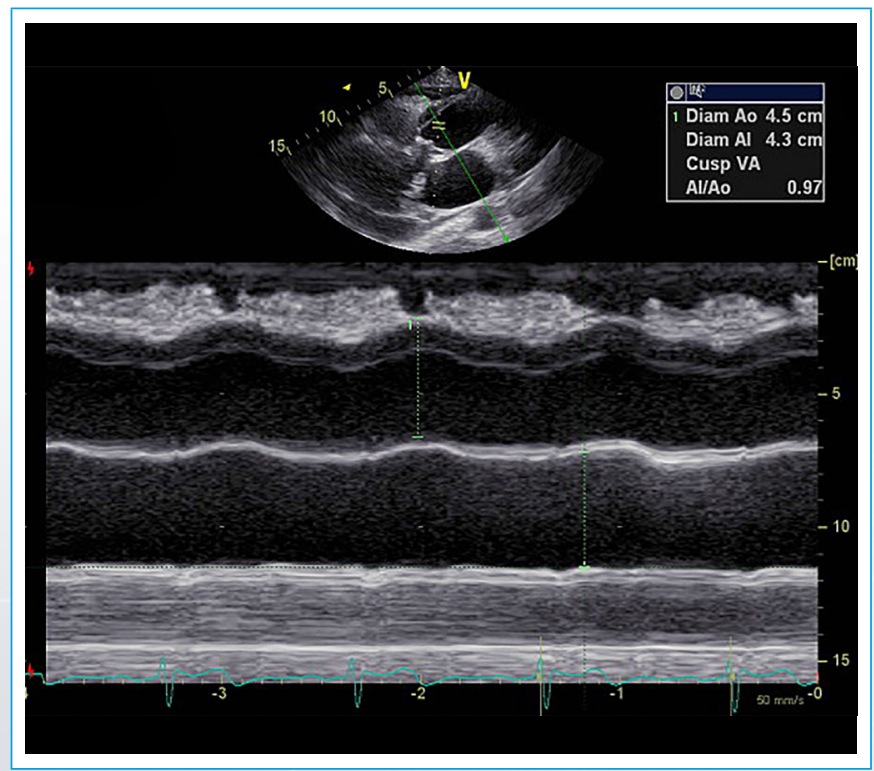

Figura 1. Hematoma intramural evaluado por modo M

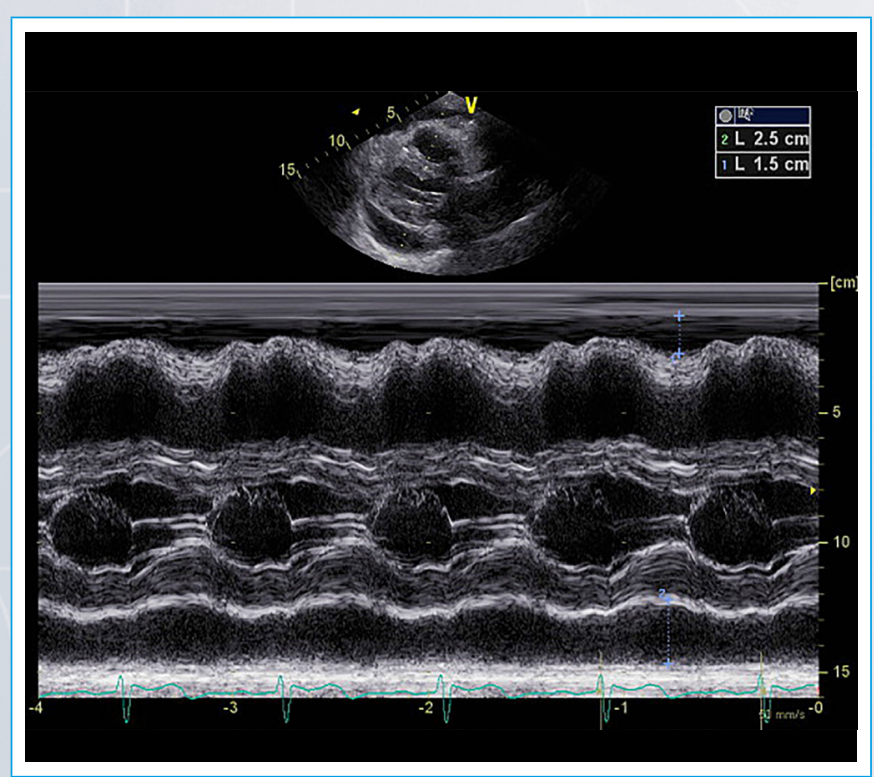

Figura 2. Modo M de ventrículos

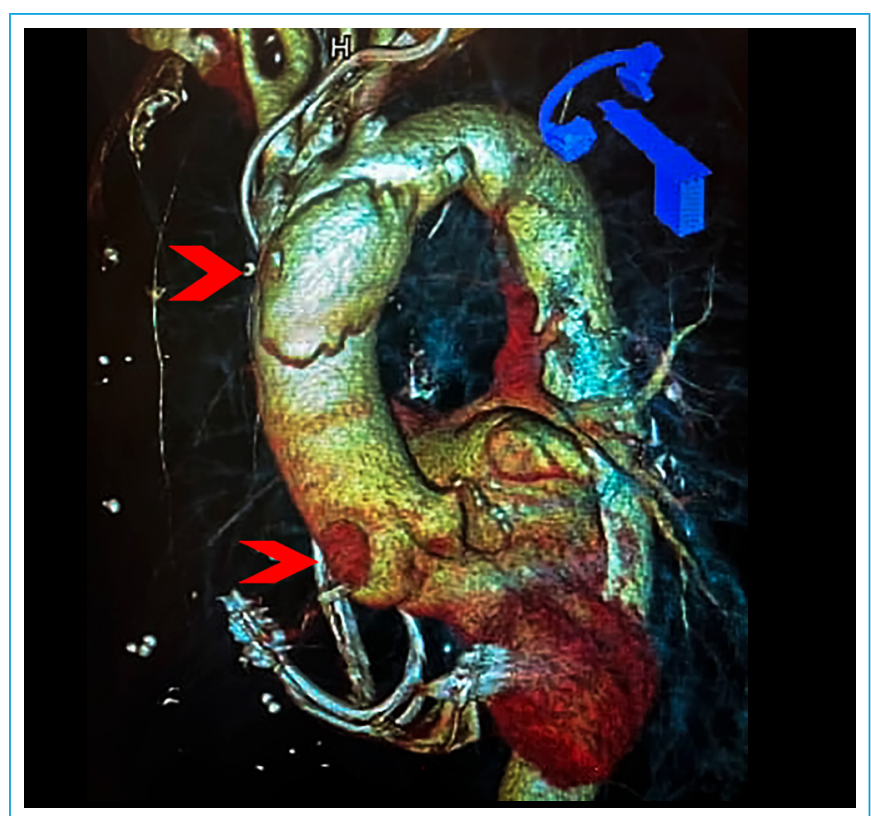

Figura 3. Imagen de TC tridimensional que muestra la aorta ascendente y las zonas de disección

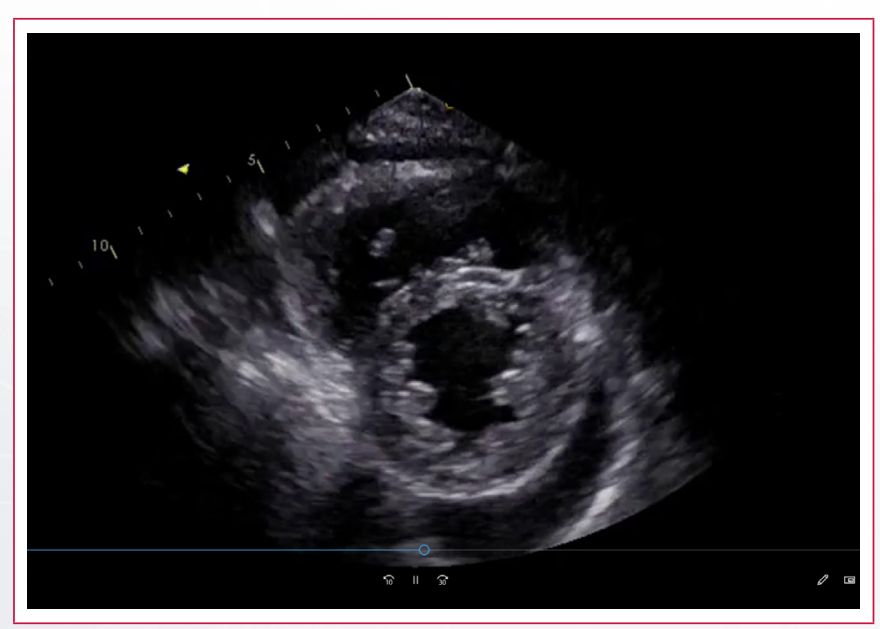

Vídeo 3. Eje corto transversal que muestra motilidad normal del ventrículo izquierdo y derrame pericárdico

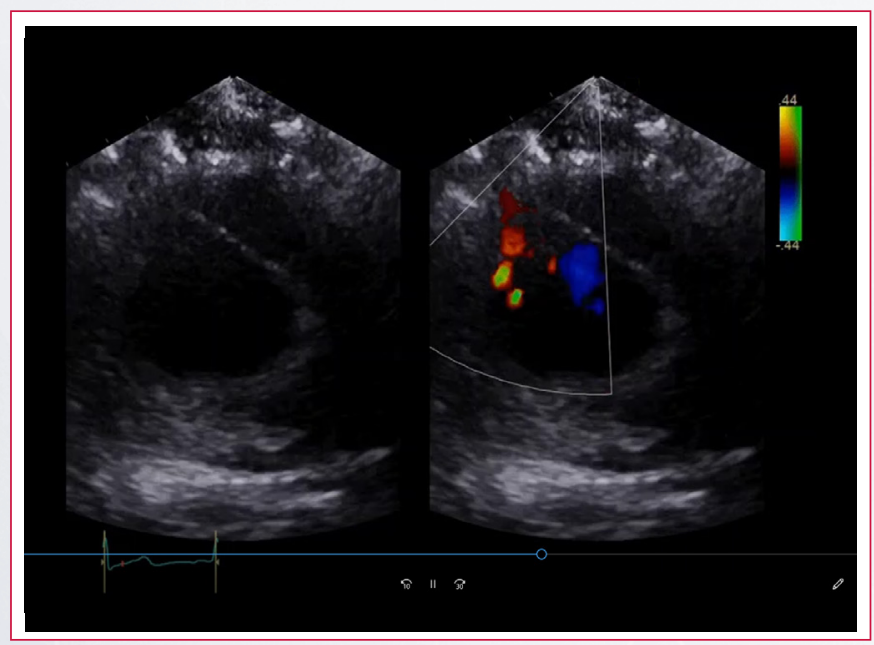

Vídeo 4. Eje corto de aorta ascendente distal desde paraesternal derecho que muestra el flap de disección 


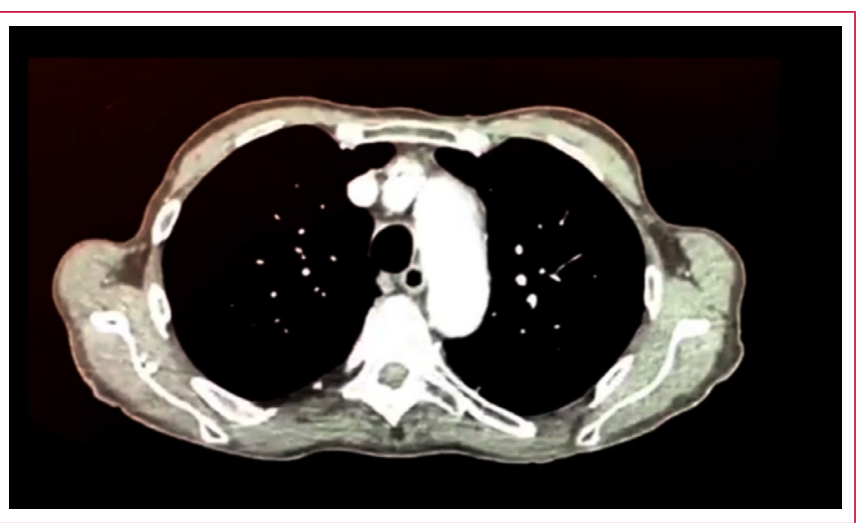

Vídeo 5. Barrido tomográfico del arco aórtico

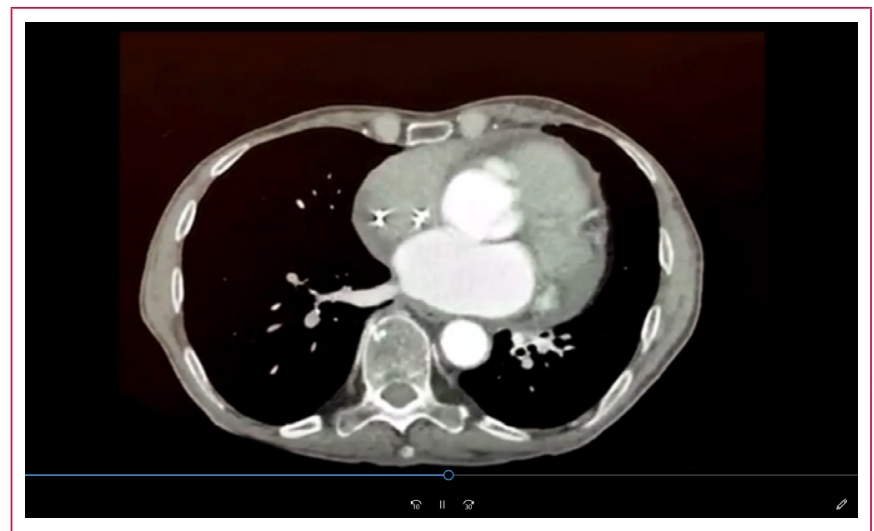

Vídeo 6. Barrido tomográfico de la raíz aórtica

\section{Discusión}

La disección de la aorta es una enfermedad grave. Se considera disección de aorta aguda en las primeras 2 semanas, subaguda de 2 semanas a 2 meses, y crónica más de 2 meses de duración ${ }^{(1)}$, pudiendo presentarse en cualquiera de sus formas: disección, hematoma intramural, disección localizada, úlcera penetrante; y pudiéndose cada una intercambiar de forma e ir hacia la ruptura o hacia la curación. En el caso de este paciente se detectó de manera asintomática con la ETT.

En casos agudos, la ETT es la primera herramienta con la que se hace frente a esta enfermedad, teniendo una alta sensibilidad que puede llegar al $85 \%$ con las mejorías de la imagen armónica y que permite evaluar además la presencia de insuficiencia aórtica, derrame pericárdico o evaluar la contractilidad del ventrículo izquierdo y la función del derecho(2). La ETT es mucho menos sensible en la disección aórtica distal tipo B ${ }^{(2)}$.

Cuando la sospecha de disección es alta y la ETT negativa, debe utilizarse otra técnica de imagen más sensible y específica. Si el paciente está estable, la resonancia magnética (RM) es la técnica de elección. En los pacientes inestables, la ecocardiografía transesofágica (ETE) puede realizarse en la cama del paciente, siendo la tomografía computarizada otra alternativa por su rapidez.

La ETE es muy precisa para los dos tipos (A y B) de disección, llegando su sensibilidad casi al 100\%, teniendo algún problema de especificidad por los artefactos que provoca el sonido(3).
La TC gatillada es una técnica muy disponible, rápida y excluye casi 100\% la presencia de disección, los pseudoflaps o artefactos por pulso ya no se ven más con la técnica gatillada y los tomógrafos modernos ${ }^{(4,5)}$.

La RM permite evaluar en integridad al paciente con disección permitiendo no sólo detectar el flap disecante, sino los puntos de entrada y reentrada, evaluar la severidad de la insuficiencia aórtica y el compromiso de arterias viscerales, con una sensibilidad y especificidad muy altas, cercanas al 100\%(6). Tiene como limitación que no siempre está disponible, los tiempos de escaneo son más largos y es más difícil monitorizar a paciente críticos en su ambiente.

El paciente de este caso presentaba 2 signos ominosos asociados a la disección aórtica, como son la insuficiencia aórtica y el derrame pericárdico, que ya estaban presentes en ecocardiografías previas. Se realizó la TC para complementar el diagnóstico ecocardiográfico por la presencia del marcapasos, que crea artefactos en la RM y por ser 100\% dependiente del mismo.

El escaneo meticuloso con ecocardiografía de la porción distal de aorta ascendente permitió detectar el flap intimal en una zona que generalmente es ciega para la ETE por interposición bronquial.

La Tabla 1 presenta datos del documento de consenso publicado en la revista de la Sociedad Americana de Ecocardiografía(7) para la evaluación de la aorta y compara las diferentes técnicas para el diagnóstico del síndrome aórtico agudo.

\begin{tabular}{|l|c|c|c|c|c|}
\hline \multicolumn{7}{|c|}{ Comparación de diferentes técnicas de diagnóstico } \\
\hline \multicolumn{1}{|c|}{ Performance } & TC & ETT & ETE & RM & Angiografía \\
\hline Sensibilidad & +++ & ++ & +++ & +++ & ++ \\
\hline Especificidad & +++ & ++ & +++ & +++ & +++ \\
\hline $\begin{array}{l}\text { Detección de } \\
\text { hematoma }\end{array}$ & +++ & + & ++ & +++ & - \\
\hline $\begin{array}{l}\text { Sitio de ruptura } \\
\text { intimal }\end{array}$ & +++ & - & ++ & +++ & ++ \\
\hline $\begin{array}{l}\text { Insuficiencia } \\
\text { aórtica }\end{array}$ & - & +++ & +++ & ++ & +++ \\
\hline $\begin{array}{l}\text { Compromiso } \\
\text { coronario }\end{array}$ & + & - & ++ & + & +++ \\
\hline $\begin{array}{l}\text { Derrame } \\
\text { pericárdico }\end{array}$ & ++ & +++ & +++ & ++ & - \\
\hline
\end{tabular}

Tabla 1. Comparativa de las diferentes técnicas diagnósticas para el síndrome aórtico agudo

Si bien no se dispone del historial clínico previo del paciente, es muy probable que el episodio inicial se tratase de un síndrome aórtico agudo y que el hematoma en la aorta ascendente haya sido iatrogénico. El riesgo de progresión de la disección o ruptura obliga al tratamiento quirúrgico de este tipo de pacientes.

\section{Conclusión}

Se ha presentado un caso de disección aórtica crónica inusual a dos niveles y con dos aspectos distintos, un hematoma intramural en aorta ascendente proximal y una disección localizada en aorta ascendente distal, no relacionados, que muestra el valor de la ETT en su evaluación, confirmándose los hallazgos porla TC. 


\section{Ideas para recordar}

- Se debe sospechar siempre un síndrome aórtico agudo en el paciente con dolor torácico y factores de riesgo (hipertensión arterial, aorta bicúspide, aspecto marfanoide, etc.), especialmente si se descarta un episodio coronario agudo.

- La ecocardiografía es la primera herramienta y se debe utilizar todas las ventanas disponibles para evaluar la mayoría de segmentos aórticos. Un estudio negativo en pacientes con alta sospecha no descarta una disección aguda.

- Por su rapidez y disponibilidad, la TC es la técnica que usualmente complementa los hallazgos ecocardiográficos.

\section{Bibliografía}

1. DeBakey ME, Beall ACJ, Cooley DA, et al. Dissecting aneurysms of the aorta. Surg Clin North Am 1966; 46: 1045-1055.

2. Cecconi M, Chirillo F, Costantini C, et al. The role of transthoracic echocardiography in the diagnosis and management of acute type A aortic syndrome. Am Heart J 2012; 163: 112-118.
3. Goldstein SA. Echocardiographic evaluation of aortic dissection. Cardiac Ultrasound Today 2001; 7: 167-180.

4. Harris KM, Strauss CE, Eagle KA, et al. Correlates of delayed recognition and treatment of acute type A aortic dissection: the International Registry of Acute Aortic Dissection (IRAD). Circulation 2011; 124: 1911-1918.

5. Shiga T, Wajima Z, Apfel CC, et al. Diagnostic accuracy of transesophageal echocardiography, helical computed tomography, and magnetic resonance imaging for suspected thoracic aortic dissection: systematic review and meta-analysis. Arch Intern Med 2006; 166: 1350-1356.

6. Nienaber CA, Spielmann RP, von Kodolitsch Y, et al. Diagnosis of thoracic aortic dissection. Magnetic resonance imaging versus transesophageal echocardiography. Circulation 1992; 85: 434-447.

7. Goldstein SA, Evangelista A, Abbara S, et al. Multimodality Imaging of Diseases of the Thoracic Aorta in Adults: From the American Society of Echocardiography and the European Association of Cardiovascular Imaging: Endorsed by the Society of Cardiovascular Computed Tomography and Society for Cardiovascular Magnetic Resonance. Journal of the American Society of Echocardiography 2015; 28 (2): 119-182. 